\title{
New Palladium-Catalyzed Reaction Pathway to Erythrina Skeleton
}

\author{
Guncheol Kim*, Jin Hee Kim and Ki Youn Lee \\ Department of Chemistry, College of Natural Science, Chungnam National University, \\ Taejon 305-764, Korea guncheol@cnu.ac.kr
}

\section{Table of Contents for Supporting Information}

\section{a. General Experimental Methods}

b. Spectral data of the following compounds and spectra

\begin{tabular}{|l|l|l|l|l|l|}
\hline Compound & ${ }^{1} \mathrm{H}$ NMR & ${ }^{13} \mathrm{C}$ NMR & IR & HRMS & Specta \\
\hline $\mathbf{1 b}$ & & & & & ${ }^{1} \mathrm{H}$ and ${ }^{13} \mathrm{C}$ NMR \\
\hline 3b & v & v & v & v & ${ }^{1} \mathrm{H}$ and ${ }^{13} \mathrm{C}$ NMR \\
\hline 4b & v & v & v & v & ${ }^{1} \mathrm{H}$ and ${ }^{13} \mathrm{C}$ NMR \\
\hline 5b & v & v & v & v & ${ }^{1} \mathrm{H}$ and ${ }^{13} \mathrm{C}$ NMR \\
\hline 6b & v & v & v & v & ${ }^{1} \mathrm{H}$ and ${ }^{13} \mathrm{C}$ NMR \\
\hline 7b & v & v & v & v & ${ }^{1} \mathrm{H}$ and ${ }^{13} \mathrm{C}$ NMR \\
\hline 8b & v & v & v & v & ${ }^{1} \mathrm{H}$ and ${ }^{13} \mathrm{C}$ NMR \\
\hline 9b & v & v & v & v & \\
\hline
\end{tabular}




\section{a. General Experimental Methods}

All reactions were performed in oven-dried glassware with magnetic stirring.

Commercial grade reagents were used without further purification. TLC analysis was performed using glass plate precoated with silica gel $60 \mathrm{~F}_{254}$. Flash column chromatography was performed with $230-400$ mesh silica gel. ${ }^{1} \mathrm{H}$ NMR spectra were obtained on $400 \mathrm{MHz}$ spectrometers. NMR spectra were recorded in parts per million ( $\delta)$ relative to the peak for tetramethylsilane $(\delta=0.00)$ as an internal standard.

\section{b. Spectral data and spectra}

Spectral data for 3b: IR (KBr): 1690, 1458, 1324, and $759 \mathrm{~cm}^{-1} ;{ }^{1} \mathrm{H}-\mathrm{NMR}$ (400 MHz, $\left.\mathrm{CDCl}_{3}\right) \delta 7.45(\mathrm{~d}, 1 \mathrm{H}, J=7.6 \mathrm{~Hz}), 7.28-7.18(\mathrm{~m}, 3 \mathrm{H}), 5.70(\mathrm{~s}, 1 \mathrm{H}), 4.88(\mathrm{~d}, 1 \mathrm{H}, J=15$ $\mathrm{Hz}), 4.34(\mathrm{~d}, 1 \mathrm{H}, J=15 \mathrm{~Hz}), 2.9-2.87(\mathrm{~m}, 1 \mathrm{H}), 2.67-2.58(\mathrm{~m}, 1 \mathrm{H}), 2.21-2.18(\mathrm{~m}, 2 \mathrm{H})$, 1.83-1.64 (m, 3H), and 1.52-1.44 (m, 1H); ${ }^{13} \mathrm{C}-\mathrm{NMR}\left(100 \mathrm{MHz}, \mathrm{CDCl}_{3}\right) \delta$ 177.5, 167.0, $141.5,141.4,128.1,126.7,123.6,122.3,119.3,77.0,47.9,40.5,28.2,27.5$, and 21.0; mass spectrum (EI) m/z $225\left(\mathrm{M}^{+}, 100\right)$; EI-HRMS : calcd for $\mathrm{C}_{15} \mathrm{H}_{15} \mathrm{NO} 225.1154$, found 225.1157 .

Spectral data for 4b: IR (KBr) : 1695, 1476, 1271, and $1037 \mathrm{~cm}^{-1}$; ${ }^{1} \mathrm{H}-\mathrm{NMR}$ (400 MHz, $\left.\mathrm{CDCl}_{3}\right) \delta 6.94(\mathrm{~s}, 1 \mathrm{H}), 6.67(\mathrm{~s}, 1 \mathrm{H}), 5.97-5.96(\mathrm{~m}, 2 \mathrm{H}), 5.73(\mathrm{~s}, 1 \mathrm{H}), 4.80(\mathrm{~d}, 1 \mathrm{H}, J=$ 
14.4 Hz), $4.27(\mathrm{~d}, 1 \mathrm{H}, J=14.4 \mathrm{~Hz}), 2.93-2.90(\mathrm{~m}, 1 \mathrm{H}), 2.61-2.58(\mathrm{~m}, 1 \mathrm{H}), 2.23-2.17(\mathrm{~m}$, 1H), 1.80-1.65 (m, 4H), and 1.50 (m, 1H); ${ }^{13} \mathrm{C}-\mathrm{NMR}\left(100 \mathrm{MHz}, \mathrm{CDCl}_{3}\right) \delta$ 177.5, 167.3, $147.8,146.7,134.5,134.3,119.4,104.1,103.3,101.4,76.7,47.9,40.6,28.2,27.4$, and 21.1; mass spectrum (EI) m/z $269\left(\mathrm{M}^{+}, 100\right)$; EI-HRMS : calcd for $\mathrm{C}_{16} \mathrm{H}_{15} \mathrm{NO}_{3} 269.1052$, found 269.1056 .

Spectral data for $\mathbf{5 b}$ : IR (KBr): 1733, 1683, 1506, 1488, 1364, 1228, and $1037 \mathrm{~cm}^{-1} ;{ }^{1} \mathrm{H}-$ NMR (400 MHz, $\left.\mathrm{CDCl}_{3}\right) \delta: 7.26(\mathrm{~s}, 1 \mathrm{H}), 7.02(\mathrm{~s}, 1 \mathrm{H}), 5.95(\mathrm{~s}, 1 \mathrm{H}), 5.92(\mathrm{~m}, 2 \mathrm{H}), 3.96$ $(\mathrm{q}, 1 \mathrm{H}, J=6.4 \mathrm{~Hz}), 3.47(\mathrm{q}, 1 \mathrm{H}, J=6.4 \mathrm{~Hz}), 2.95-2.92(\mathrm{~m}, 3 \mathrm{H}), 2.81(\mathrm{~m}, 1 \mathrm{H}), 2.42-2.39$ $(\mathrm{m}, 1 \mathrm{H}), 2.17(\mathrm{~m}, 1 \mathrm{H}), 1.68(\mathrm{~m}, 2 \mathrm{H})$, and 1.56-1.42 (m, 2H).; ${ }^{13} \mathrm{C}-\mathrm{NMR}(100 \mathrm{MHz}$, $\left.\mathrm{CDCl}_{3}\right) \delta 179.7,164.0,146.2,145.5,130.9,128.1,121.3,109.5,106.1,101.0,66.7$, 41.4, 36.6, 29.5, 28.6, 27.9, and 21.2; mass spectrum (EI) m/z $283\left(\mathrm{M}^{+}, 100\right)$; EIHRMS : calcd for $\mathrm{C}_{17} \mathrm{H}_{17} \mathrm{NO}_{3} 283.1208$, found 283.1205.

Spectral data for $\mathbf{6 b}$ : IR (KBr): 1685, 1502, and $1207 \mathrm{~cm}^{-1} ;{ }^{1} \mathrm{H}-\mathrm{NMR}\left(400 \mathrm{MHz}, \mathrm{CDCl}_{3}\right)$ $\delta 6.97(\mathrm{~s}, 1 \mathrm{H}), 6.75(\mathrm{~s}, 1 \mathrm{H}), 5.73(\mathrm{~s}, 1 \mathrm{H}), 4.85(\mathrm{~d}, 1 \mathrm{H}, J=14.4 \mathrm{~Hz}), 4.32(\mathrm{~d}, 1 \mathrm{H}, J=14.4$ Hz), $3.90(\mathrm{~s}, 3 \mathrm{H}), 3.86(\mathrm{~s}, 3 \mathrm{H}), 2.95-2.93(\mathrm{~m}, 1 \mathrm{H}), 2.61(\mathrm{~m}, 1 \mathrm{H}), 2.24-2.21(\mathrm{~m}, 2 \mathrm{H})$, 1.82-1.72 (m, 3H), and $1.53(\mathrm{~m}, 1 \mathrm{H}) ;{ }^{13} \mathrm{C}-\mathrm{NMR}\left(100 \mathrm{MHz}, \mathrm{CDCl}_{3}\right) \delta$ 177.6, 167.3, $149.6,148.0,133.4,133.3,119.3,106.5,106.3,77.2,56.4,56.0,48.0,40.7,28.4,27.5$, and 21.4; mass spectrum (EI) m/z $285\left(\mathrm{M}^{+}, 100\right)$; EI-HRMS : calcd for $\mathrm{C}_{17} \mathrm{H}_{19} \mathrm{NO}_{3}$ 
285.1365, found 285.1361.

Spectral data for $7 \mathbf{b}$ : IR (KBr): 1653, 1513, 1257, and $1223 \mathrm{~cm}^{-1} ;{ }^{1} \mathrm{H}-\mathrm{NMR}(500 \mathrm{MHz}$, $\left.\mathrm{CDCl}_{3}\right) \delta 7.02(\mathrm{~s}, 1 \mathrm{H}), 6.66(\mathrm{~s}, 1 \mathrm{H}), 5.86(\mathrm{~s}, 1 \mathrm{H}), 4.04(\mathrm{q}, 1 \mathrm{H}, J=6.5 \mathrm{~Hz}), 3.83(\mathrm{~s}, 3 \mathrm{H})$, $3.81(\mathrm{~s}, 3 \mathrm{H}), 3.38(\mathrm{q}, 1 \mathrm{H}, J=6.5 \mathrm{~Hz}), 2.96-2.77(\mathrm{~m}, 4 \mathrm{H}), 2.39-2.36(\mathrm{~m}, 1 \mathrm{H}), 2.19-2.17$ $(\mathrm{m}, 2 \mathrm{H}), 1.72-1.68(\mathrm{~m}, 1 \mathrm{H})$, and 1.51-1.37 (m, 2H). ${ }^{13} \mathrm{C}-\mathrm{NMR}\left(125 \mathrm{MHz}, \mathrm{CDCl}_{3}\right) \delta$ $169.9,164.4,147.9,146.4,129.8 .127 .0,121.0,112.3,109.8,66.2,56.2,55.7,41.5$, 36.1, 29.4, 28.2, 28.0, and 21.2; mass spectrum (EI) m/z $299\left(\mathrm{M}^{+}, 100\right)$; EI-HRMS: calcd for $\mathrm{C}_{18} \mathrm{H}_{21} \mathrm{NO}_{3} 299.1521$, found 299.1521.

Spectral data for $8 b$ : IR : $1668 \mathrm{~cm}^{-1}, 1496 \mathrm{~cm}^{-1}, 1257 \mathrm{~cm}^{-1}$, and $1039 \mathrm{~cm}^{-1}$; ${ }^{1} \mathrm{H}-\mathrm{NMR}$ (400 $\left.\mathrm{MHz}_{\mathrm{CDCl}}\right) \delta 7.43(\mathrm{~d}, 1 \mathrm{H}, J=8.4 \mathrm{~Hz}), 6.75-6.73(\mathrm{~m}, 2 \mathrm{H}), 5.90(\mathrm{~s}, 1 \mathrm{H}), 4.04(\mathrm{q}, 1 \mathrm{H} J$ $=6.6 \mathrm{~Hz}), 3.79(\mathrm{~s}, 3 \mathrm{H}), 3.49(\mathrm{q}, 1 \mathrm{H}, J=6.6 \mathrm{~Hz}), 3.01-2.85(\mathrm{~m}, 4 \mathrm{H}), 2.41-2.38(\mathrm{~m}, 1 \mathrm{H})$, $2.18(\mathrm{~m}, 1 \mathrm{H}), 1.69(\mathrm{~m}, 2 \mathrm{H})$, and 1.56-1.48 (m, 2H); ${ }^{13} \mathrm{C}-\mathrm{NMR}\left(100 \mathrm{MHz}, \mathrm{CDCl}_{3}\right) \delta$ $170.0,164.5,158.2,136.0,130.1,126.3,120.8,114.6,111.1,66.4,55.2,41.5,36.3,29.4$, 28.9, 28.0, and 21.1; mass spectrum (EI) m/z $269\left(\mathrm{M}^{+}, 100\right)$; EI-HRMS : calcd for $\mathrm{C}_{17} \mathrm{H}_{19} \mathrm{NO}_{2} 269.1416$, found 269.1415.

Spectral data for 9b: IR (KBr) : 1734, 1616, 1558, 1362, 1216 and $744 \mathrm{~cm}^{-1}$; ${ }^{1} \mathrm{H}-\mathrm{NMR}$ $\left(400 \mathrm{MHz}, \mathrm{CDCl}_{3}\right) \delta: 7.00(\mathrm{~s}, 1 \mathrm{H}), 6.70(\mathrm{~s}, 1 \mathrm{H}), 5.96(\mathrm{~s}, 1 \mathrm{H}), 4.10(\mathrm{~m}, 1 \mathrm{H}), 4.04(\mathrm{~m}$, 4H), 3.85 (s, 3H), $3.75(\mathrm{~s}, 3 \mathrm{H}), 3.57(\mathrm{~m}, 1 \mathrm{H}), 2.98(\mathrm{~m}, 1 \mathrm{H}), 2.42-2.30(\mathrm{~m}, 4 \mathrm{H}), 1.95-$ 
1.87(m, 1H), and 1.28-1.24(m, 2H); ${ }^{13} \mathrm{C}-\mathrm{NMR}\left(100 \mathrm{MHz}, \mathrm{CDCl}_{3}\right) \delta: 172.2,163.6$, $159.4,148.1,146.6,128.7,126.5,115.0,112.0,108.4,95.4,69.5,64.5,60.7,55.9,55.8$, 37.3, 33.4, 27.0, and 26.9; mass spectrum (EI) m/z $357\left(\mathrm{M}^{+}, 100\right)$; EI-HRMS : calcd for $\mathrm{C}_{20} \mathrm{H}_{23} \mathrm{NO}_{5} 357.1576$, found 357.1581. 


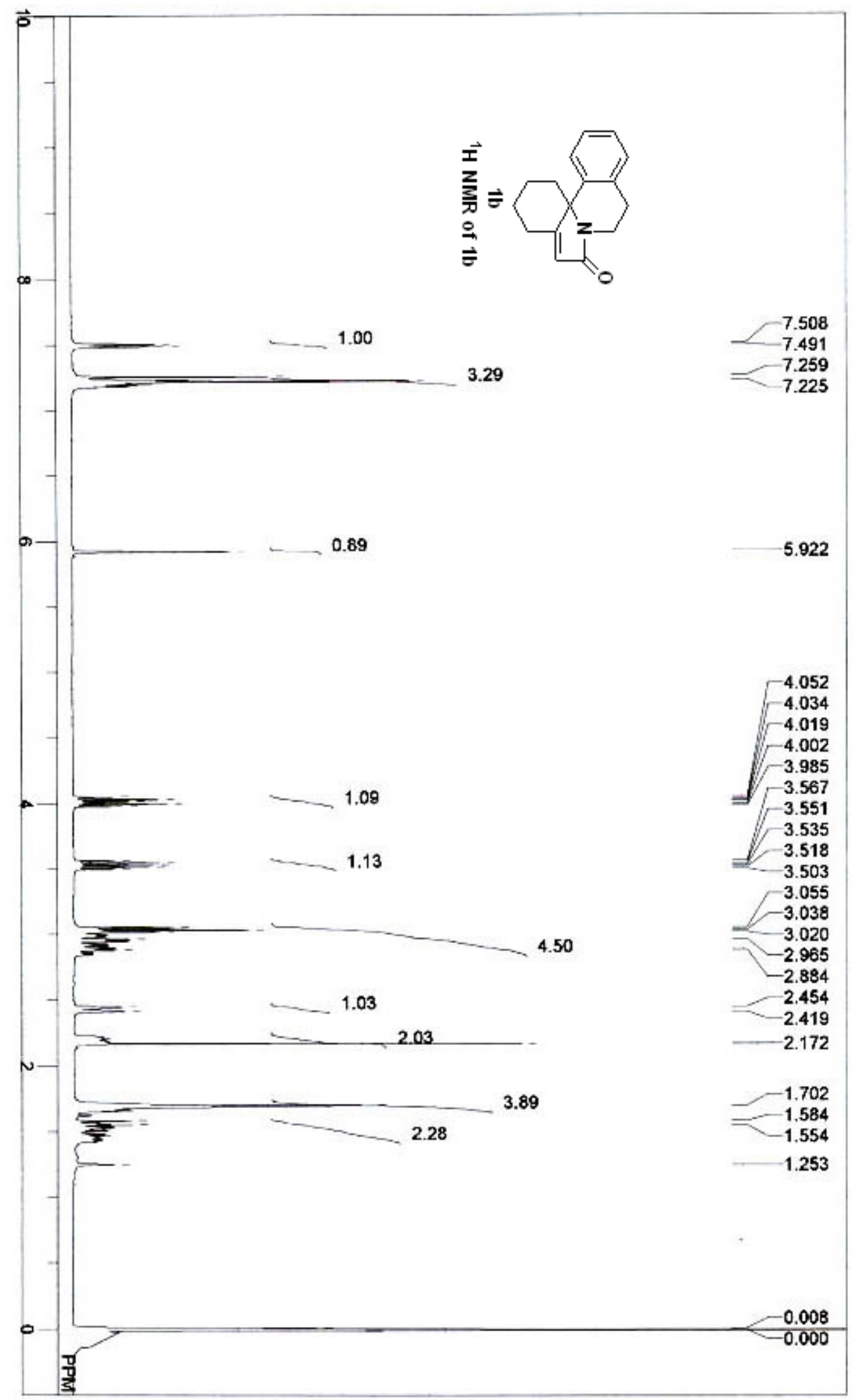




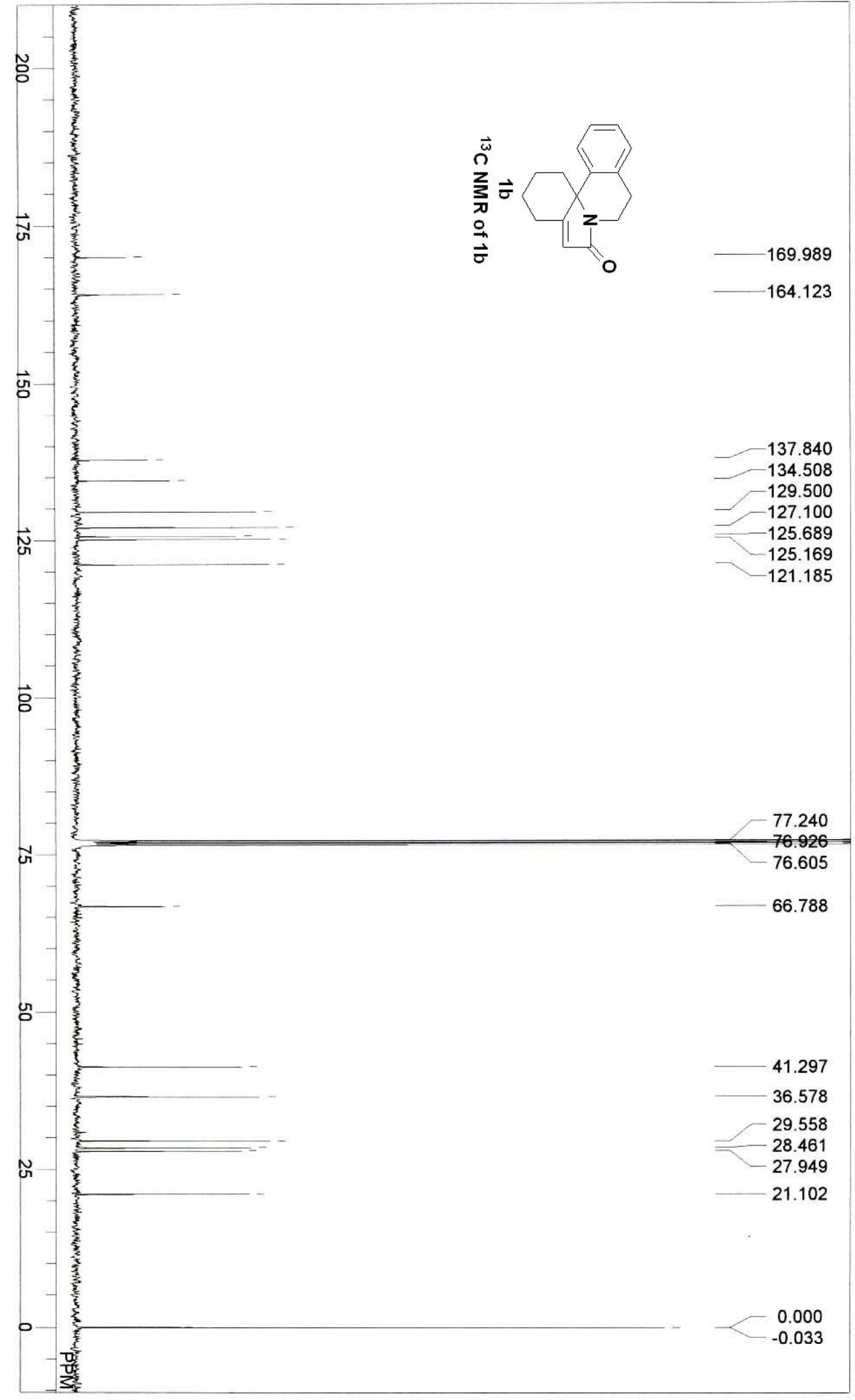




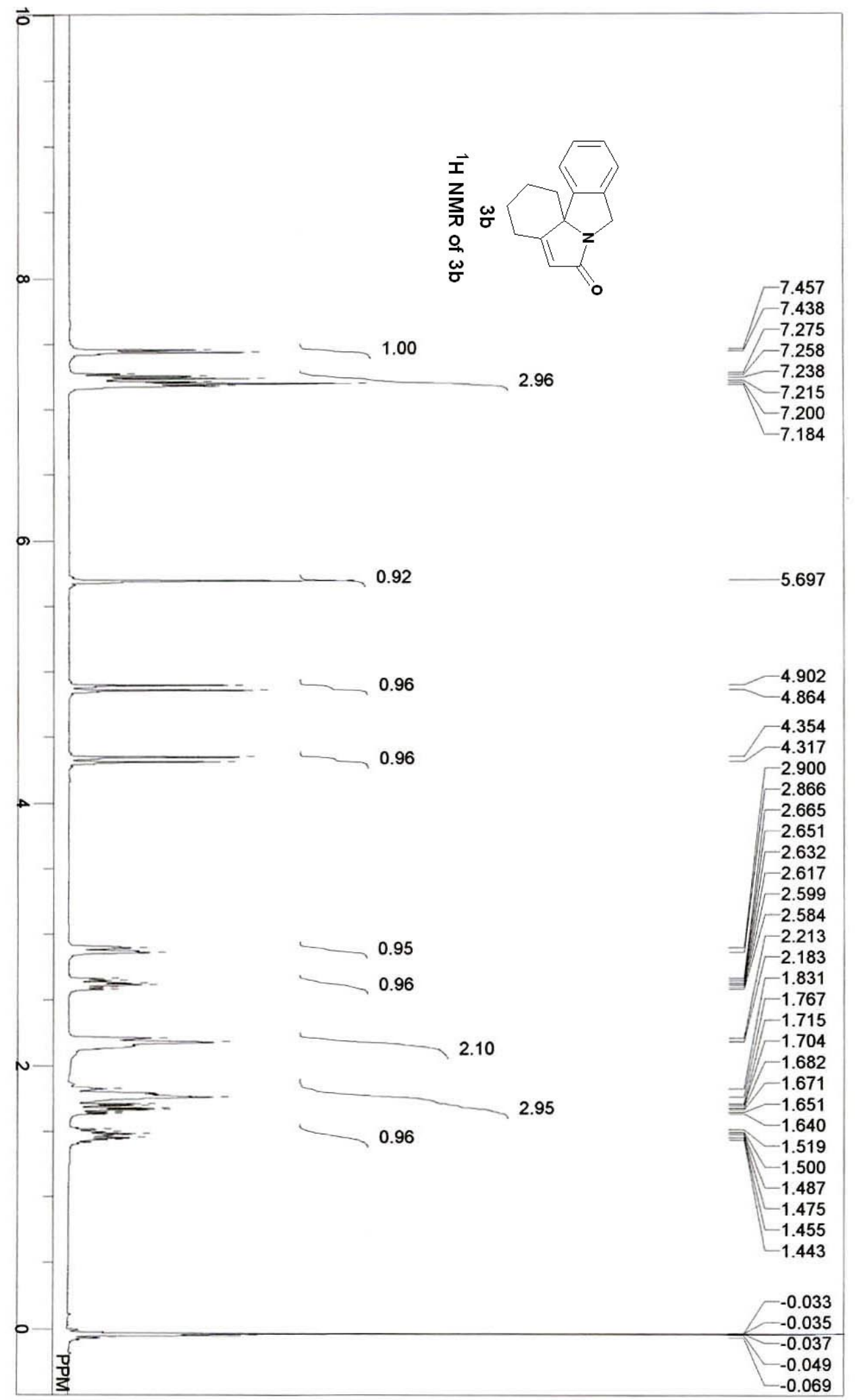




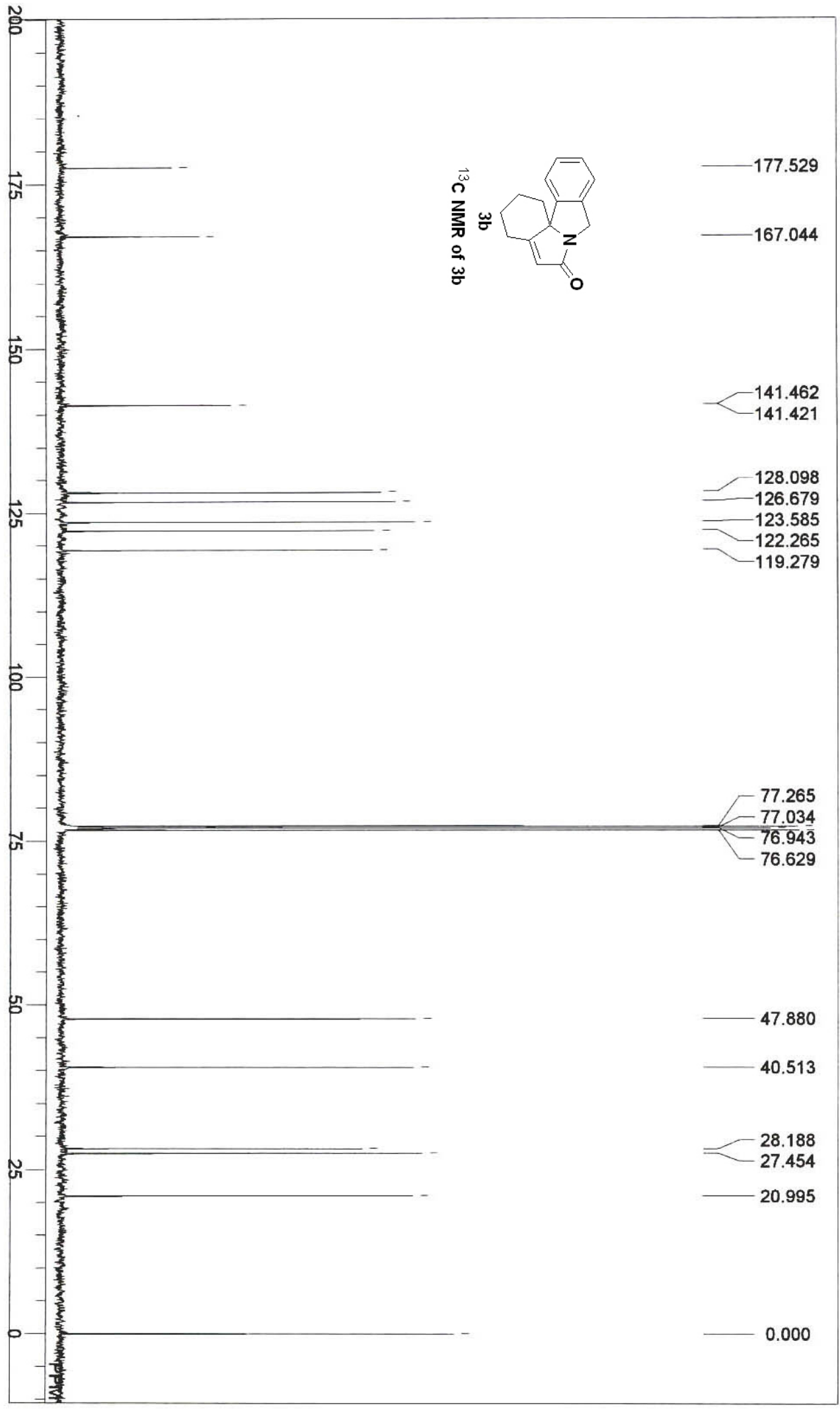




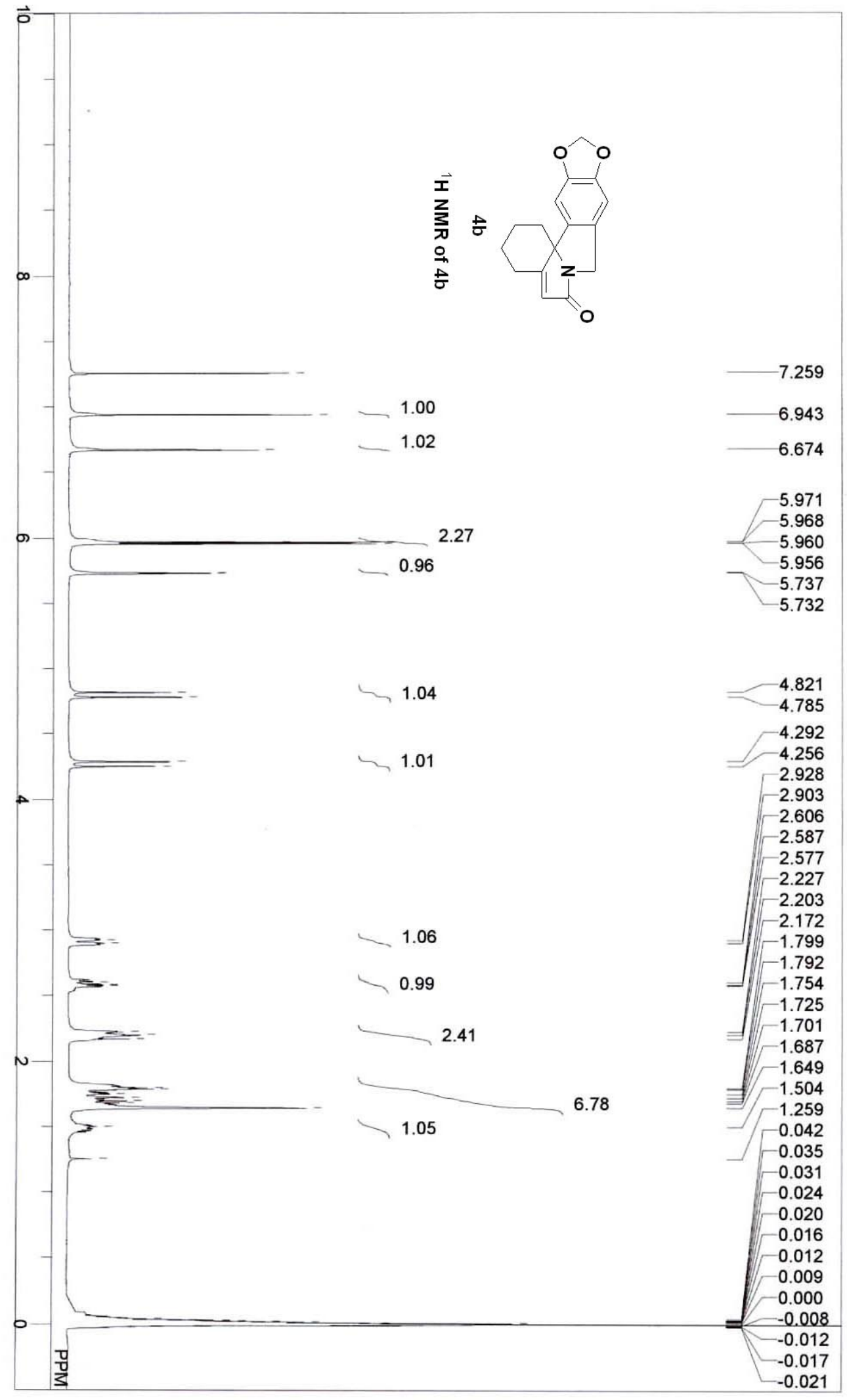




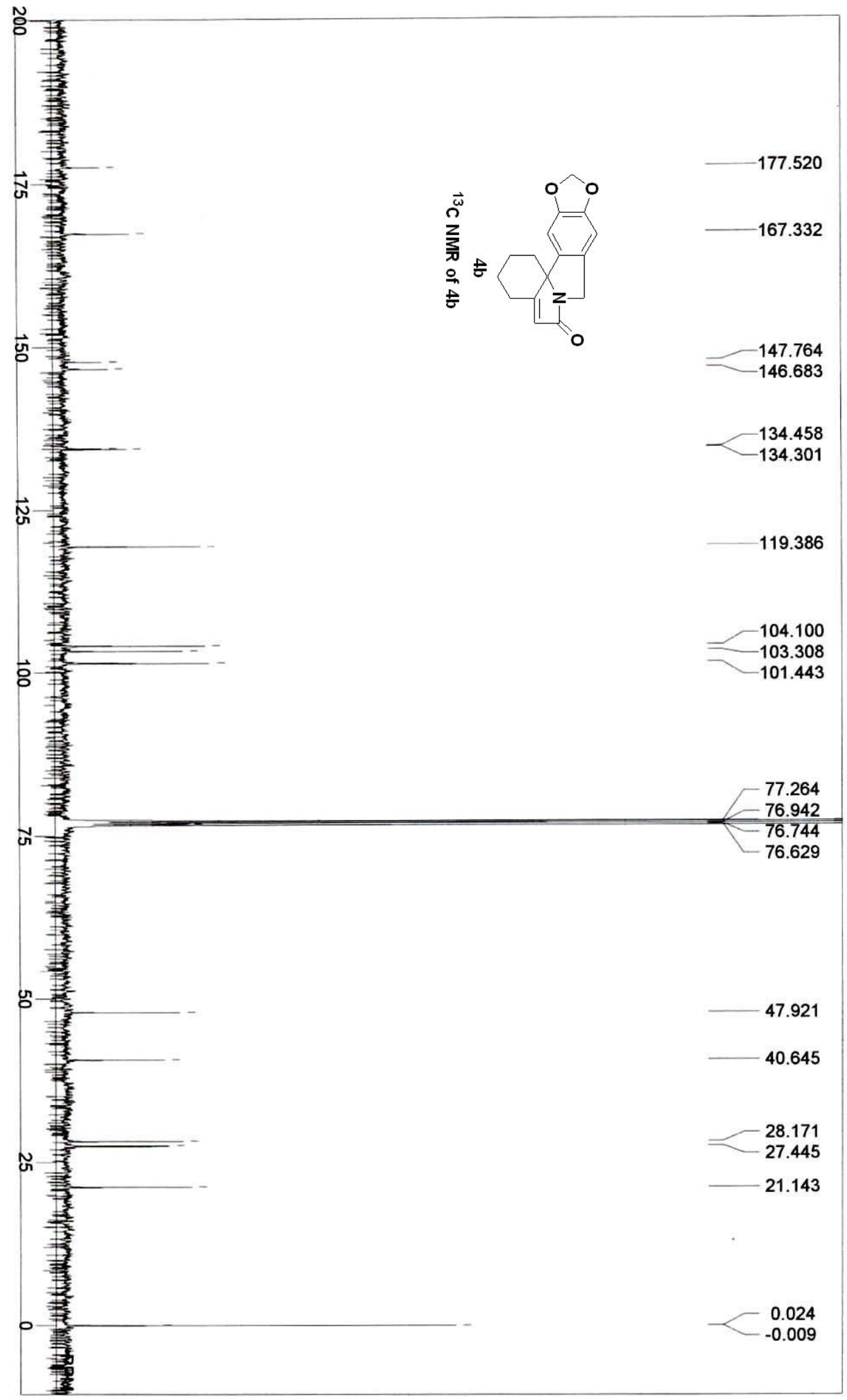




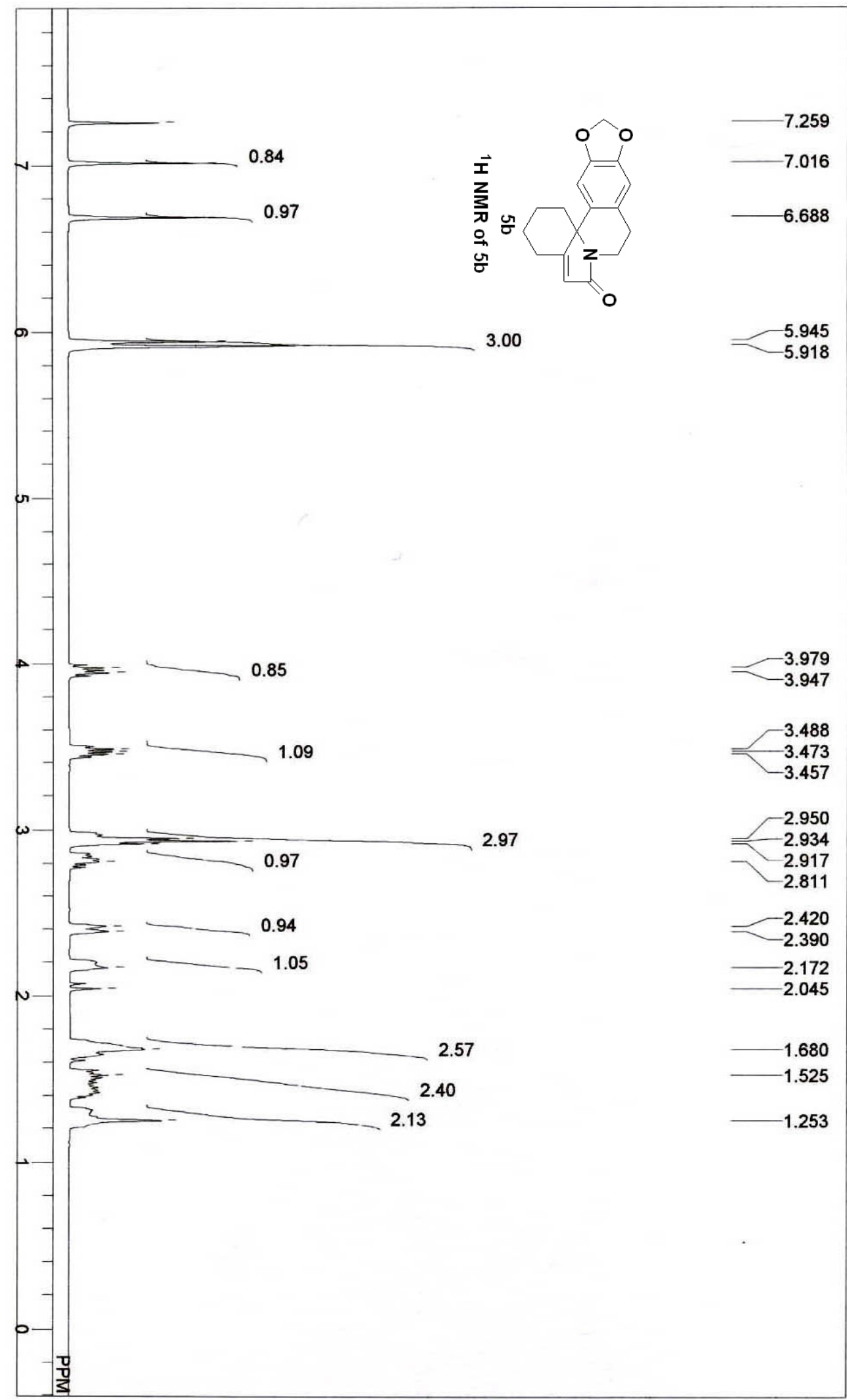




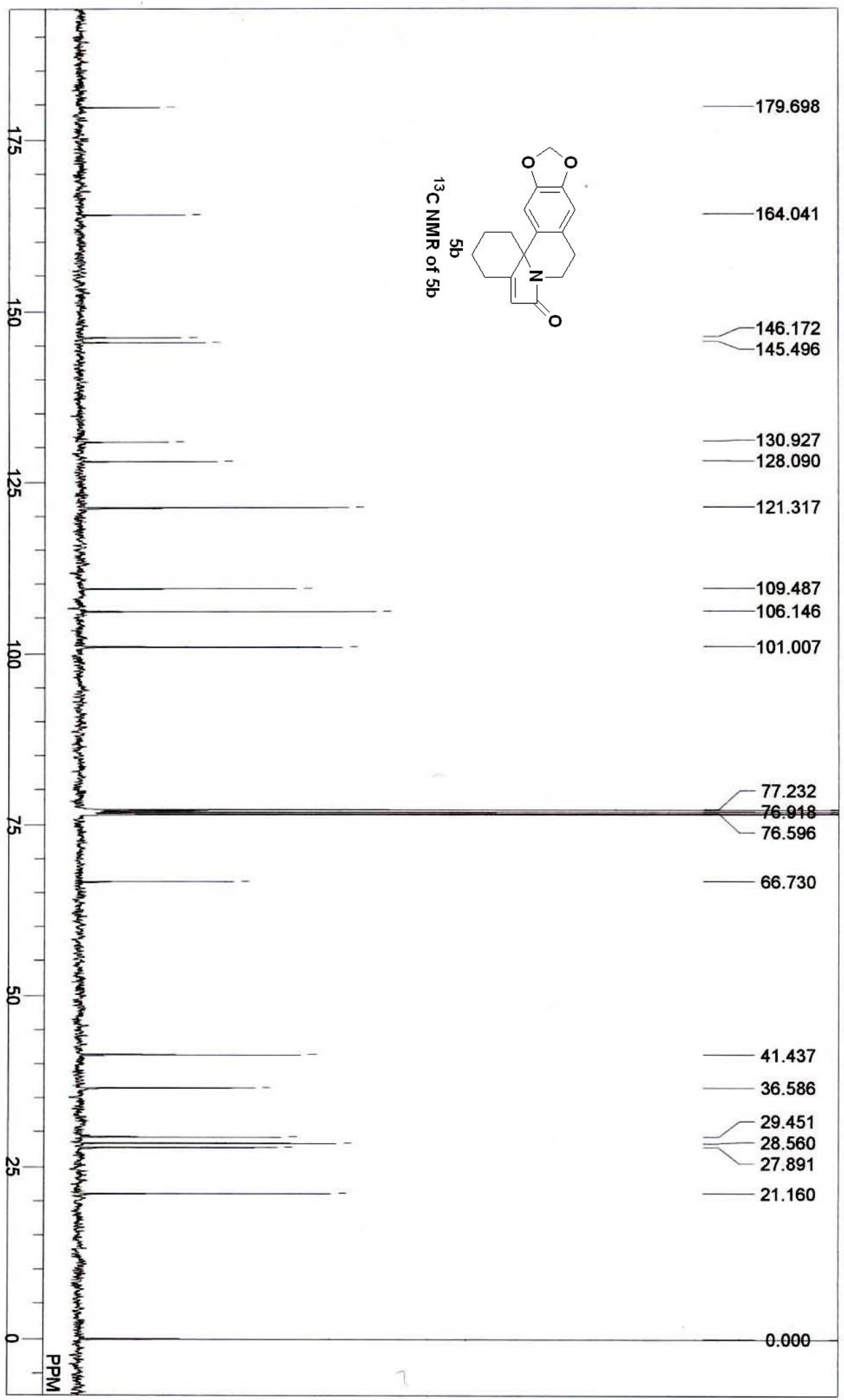




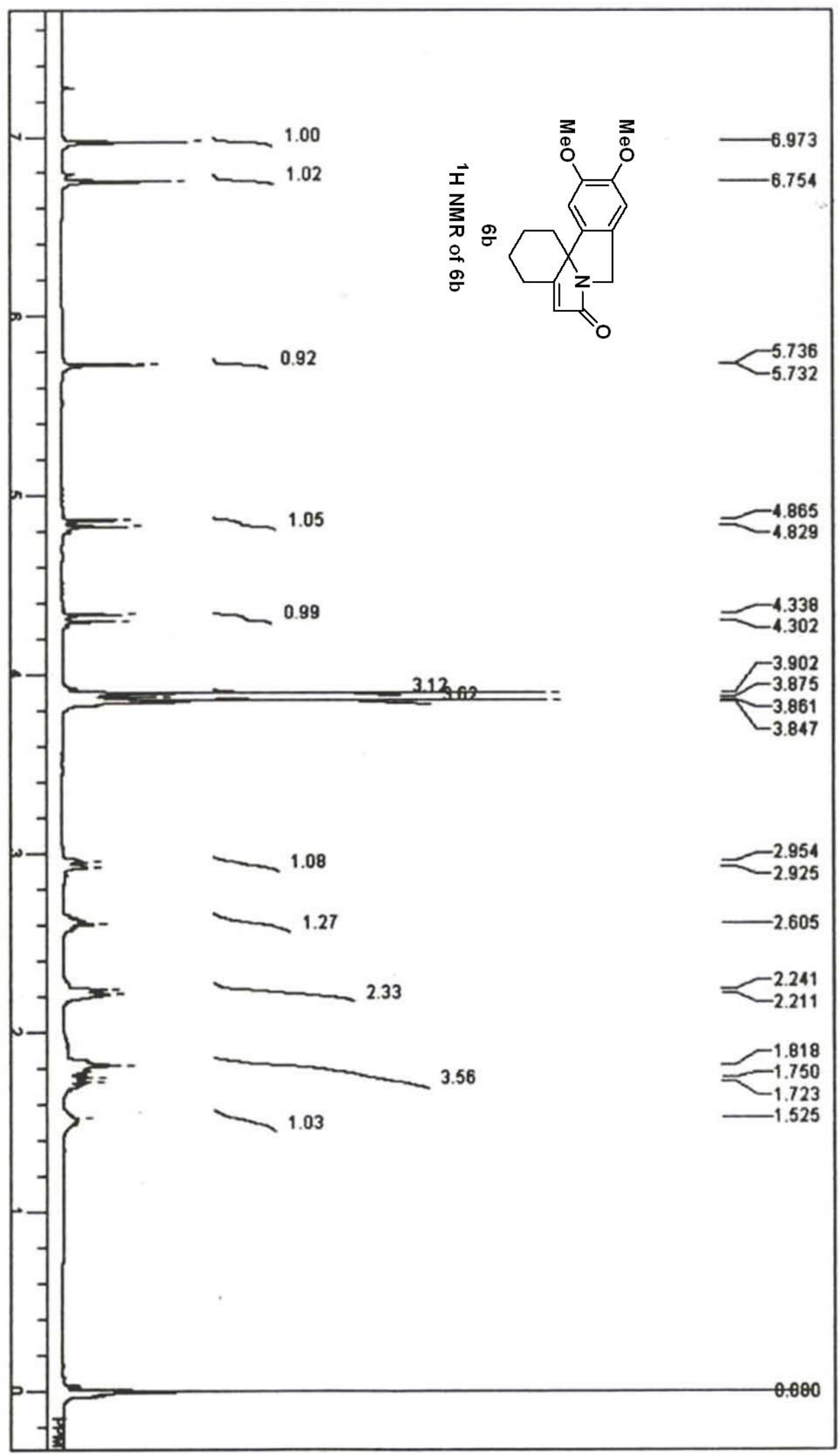




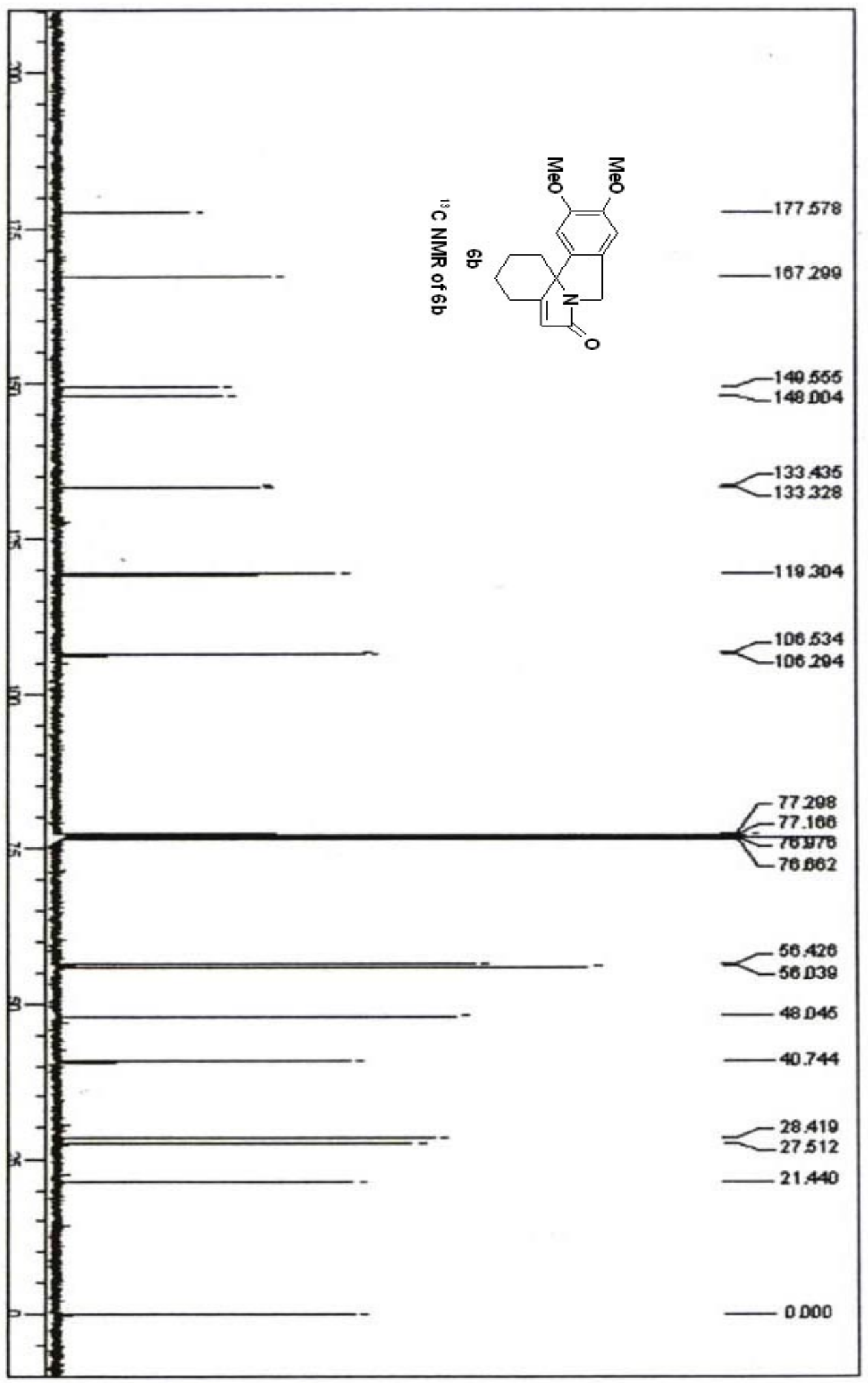




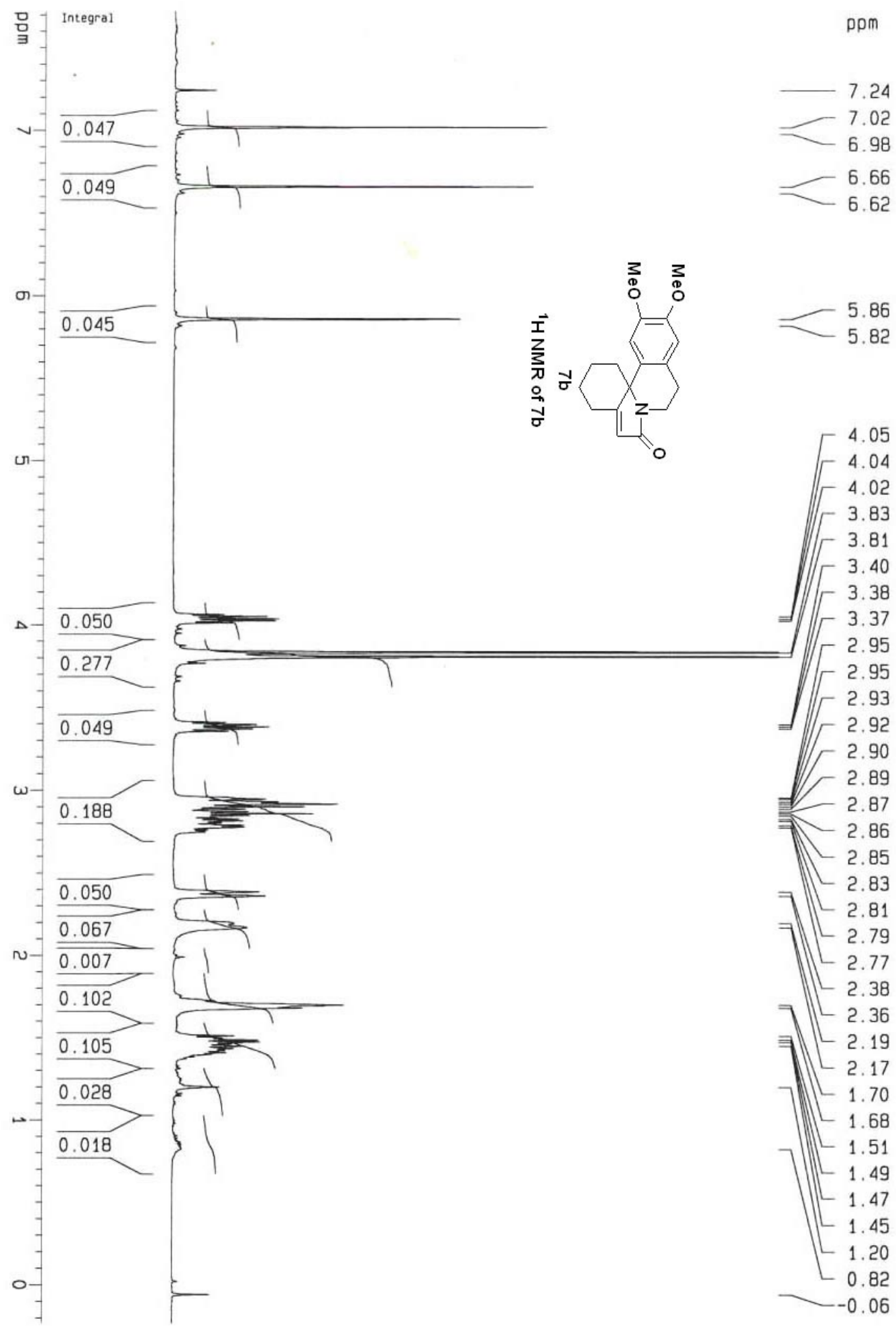




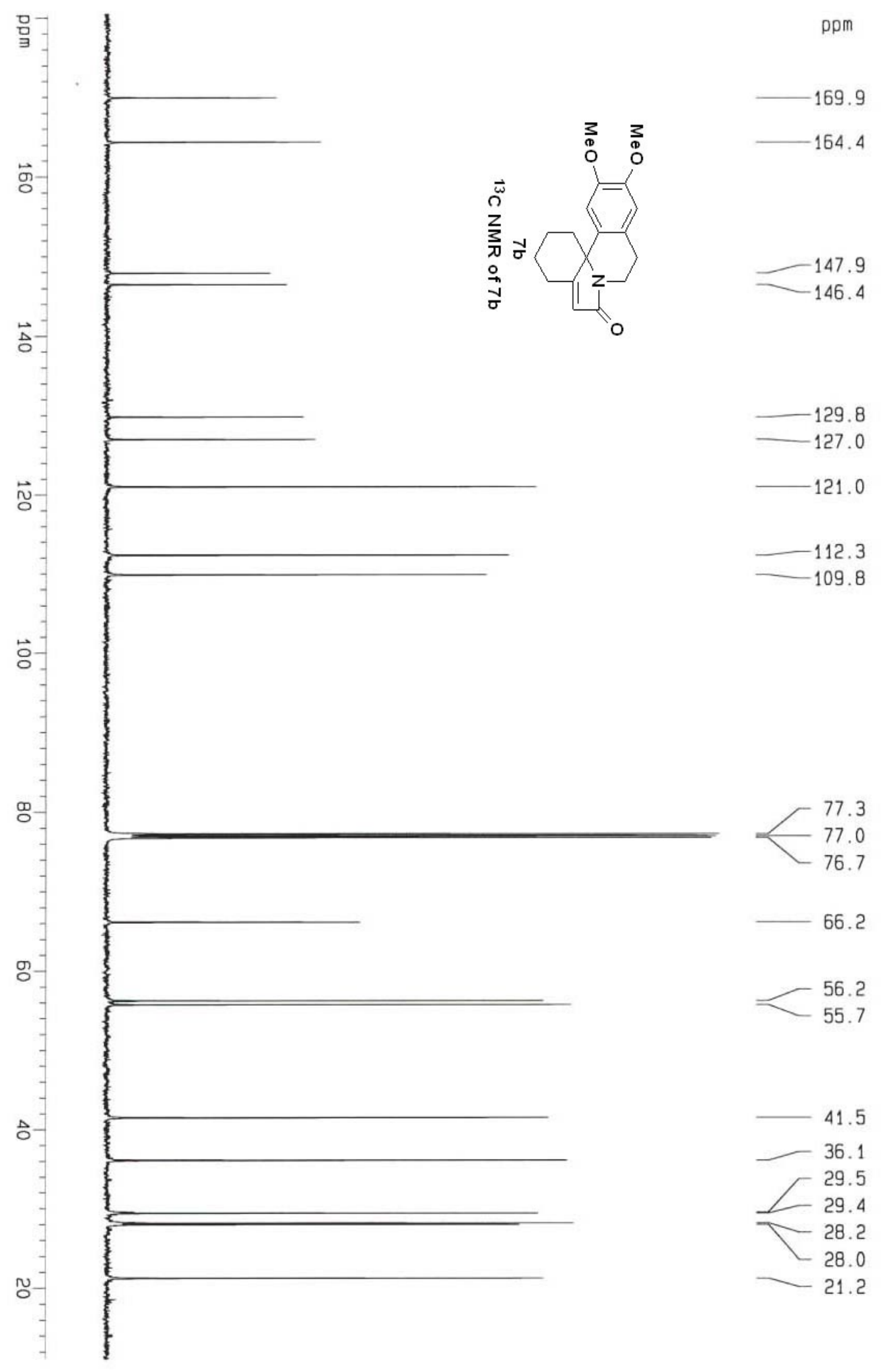




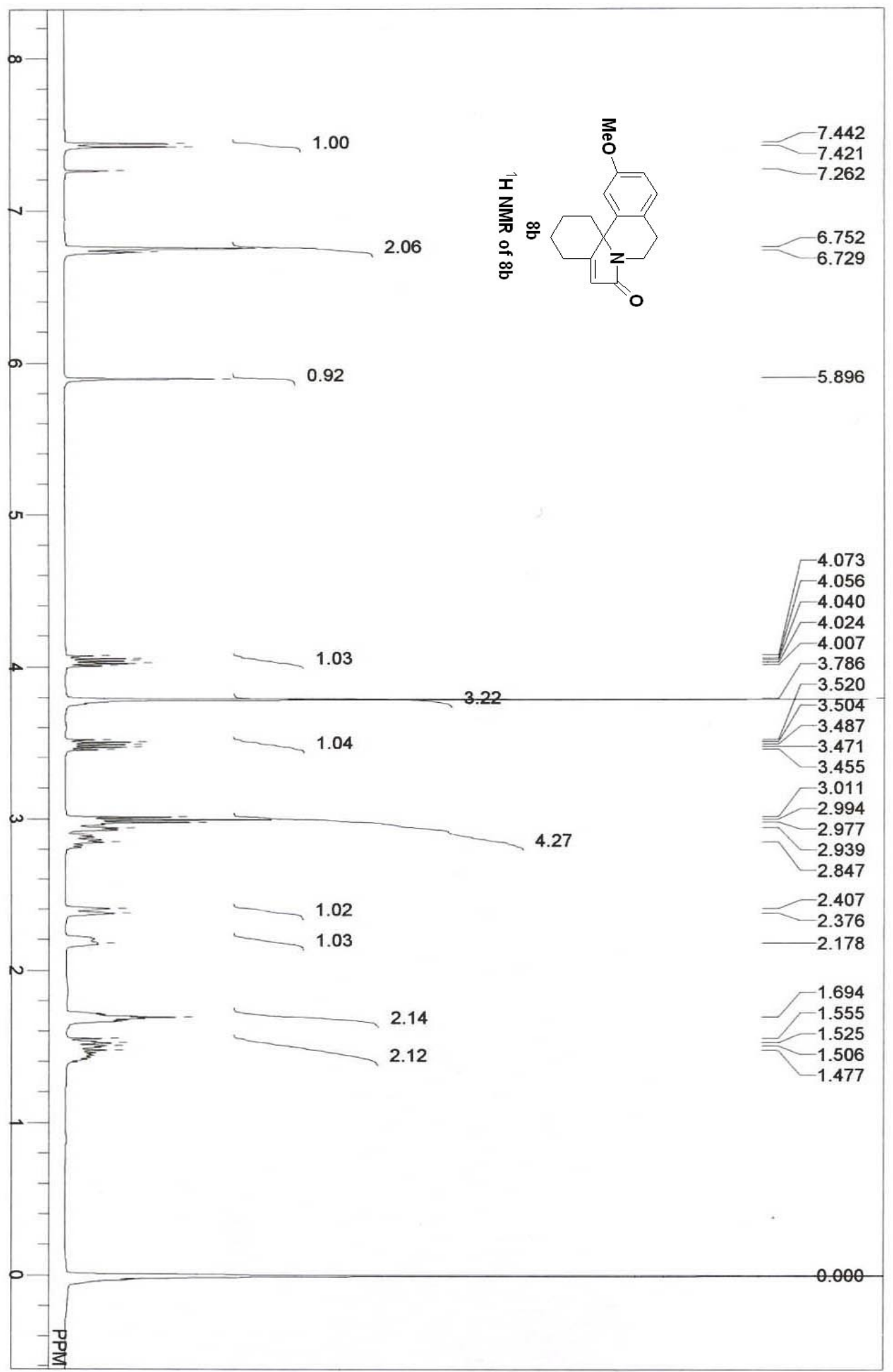




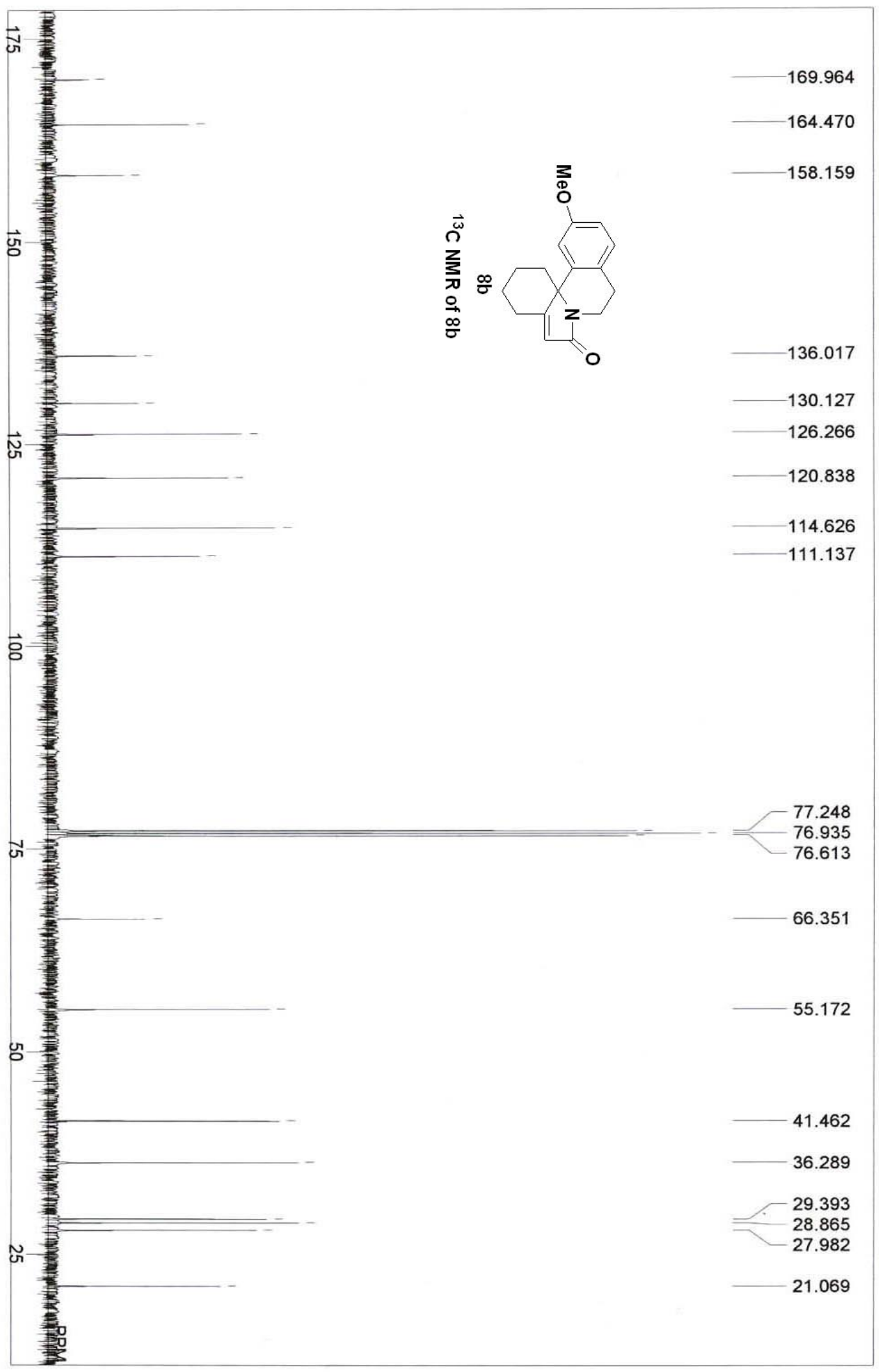

